\title{
Study on the Operating Room Occupational Safety and Radiation Protection Management
}

\author{
Changxia Cheng, Huan Zhang, Linlin Li
}

Sino-Japanese Friendship Hospital, Jilin University, Changchun, Jilin, 130033

Keywords: Radiation Protection Management, Occupational Safety, Protection Method

\begin{abstract}
This paper analyzes the current situation of radiological protection in the operating room of medical institutions, and puts forward some countermeasures for existing defects in the current situation. Questionnaire was issued to the operating rooms of several medical institutions that carried out intraoperative radiation and the actual status of intraoperative radiological protection was obtained. According to results, in the operation rooms of 21 medical institutions, there were 24 mobile radiology equipment in 19 medical institutions operating rooms and 14 radiological protective clothing in the operating rooms of the medical institutions. Only $7.37 \%$ of nursing staffs were identified as wearing protective clothing during operation, $28.42 \%$ of nurses did not wear protective clothing during operation, and more than $60 \%$ of nurses did not wear protective clothing while performing radiological operations, and left the operating room or stood behind the protective screen to avoid the radiation. Nurses in all operating rooms surveyed have not received any professional training in radiological protection. Therefore, Intraoperative radiological protection facilities in medical institutions are equipped and used more freely. The lack of specific knowledge of operatives and improper protection methods should be paid more attention and improvement.
\end{abstract}

\section{Introduction}

Since Roentgen found X-ray more than 100 years ago, X-ray has been used to diagnose and treat human diseases by penetrating the surface and seeing another change of human internal structure. For more than 100 years, great changes have taken place in X-ray equipment, from the simplest $\mathrm{X}$-ray device to digitized computerized equipment. The birth of mobile C-arm and G-arm X-ray machines has provided the possibility of accurate positioning during the operation, so it is widely used in the operating room. However, the operating room staff is not a professional radiation practitioners, limited understanding of the X-ray, resulting in some operating room staff too neglect and ignore the dangers of lack of protection awareness, resulting in poor protection compliance. And some other operating room staff over-fear, enlarge its harmfulness, that X-ray on the human body is harmful, should try to avoid contact, unwilling to do such surgery, or excessive protection and affect the quality of work. In response to these circumstances, the author of the operating room $\mathrm{X}$-ray safety protection situation to investigate the appropriate X-ray safety strategy to protect medical staff and patient safety and clinical quality.

\section{Objects and Methods}

A total of 37 operating room nurses who participated in the training in Hong Kong after their assessment came from 37 hospitals in Guangdong Province. Each hospital selected one operating room specialist nurse. Including 17 in Guangzhou City, 6 in Shenzhen City and 2 in Zhuhai City, and 1 in 12 other cities in the vicinity; 26 from Grade Three First Class Hospitals, seven Grade Two Class A hospitals and four others. 37 operating room specialist nurses aged 19 to 42 years old, 36 females, 1 males, working years of 3 to 22 years, specialist work time are more than 3 years, to understand where the hospital's X-ray protection.

Under the guidance of relevant experts, the questionnaire should be designed by ourselves, including five dimensions: (1) the basic information of the respondent; (2) the cognition and training of operating room X-ray protection; (3) the X-ray protective equipment and environment 
Management; (4) relevant system management and guidance; (5) X-ray protection during operation and practice. Reliability analysis of the questionnaire using SPSS 13.0 statistical software, the five dimensions of the internal consistency reliability coefficient Cronbach's $\alpha$ are: $0.841,0.832,0.841,0.851,0.812$; external reliability through the same group of 15 days after the survey object weight Test, retest reliability 0.832 , indicating that the reliability of the questionnaire.

Questionnaires were distributed anonymously. On the same day, 37 questionnaires were distributed and 37 questionnaires were returned, with a recovery rate of $100 \%$.

\section{Data Analysis}

Of the 37 operating room specialist nurses, only 10 considered knowledge about X-ray protection, and 27 nurses preferred or did not understand it. Operating room nurses on X-ray protection-related knowledge and lack of knowledge about the lack of training in their hospitals. Of the 37 hospitals surveyed, only five hospitals regularly organized relevant trainings, 12 hospitals organized occasional training and 20 hospitals had never organized training. Lack of awareness and training, resulting in nurses on the X-ray protection misconduct. The survey shows: There are $22 \%$ of operating room nurses do not use protection, that every trouble wearing lead clothing, airtight, too heavy, easy to cause muscle fatigue, strain, poor protection compliance. The hospital and department should regularly let the operating room nurses receive X-ray radiation safety training, including X-ray basic knowledge, radiation safety, biological effects of radiation, effective protective measures and so on. Responsible for the establishment of protection responsible for annual updates of radiation protection related content, testing the integrity of protective equipment to ensure its protective properties. Excessive fear of staff, in addition to strengthening continuing education, regular training in radiation protection knowledge. The radiation dose can be applied to the personal monitor monitor staff, with the actual results for the staff to dispel doubts, to explain to employees through the appropriate protection, X-ray Human body without damage.

Of the 37 hospitals, 11 hospitals did not have a special operating room to prevent X-ray transmission. 18 hospitals lacked protective equipment but did not have enough protective equipment. Four hospitals did not have any protective equipment. There were no warning signs hanging in the X-ray films of 17 hospital operating rooms. This shows that there are still some facilities for protection of hospitals that are still imperfect in supporting facilities for the protection of the hospitals. As a result, the environment is not safe, environmental requirements should be raised, special operating rooms should be set up and warning systems should be set up to promptly notify employees when using radiation. The best application of radiation protection walls, walls and doors and windows are required to install protective layer. Should choose a larger operating room to place mobile $\mathrm{C}$ arm machine, placed $\mathrm{C}$ arm machine operating room area should not be less than 24 $\mathrm{m} 2$. The closer the C-arm machine is to the wall, the more reflection and scattering lines will be produced by the wall. Use the C-arm as far as possible to extend the distance from the wall behind the operating room. Strengthen mechanical ventilation to reduce the concentration of harmful substances such as nitrogen oxides and ozone in the operating room and maintain the balance of the negative ions for download and the appropriate microclimate. Surgical items should be neat and tidy, should not be placed too many items. Too many items will cause X-ray scattering of the refraction, resulting in secondary rays.

Among the 37 hospitals, 18 hospitals did not have any guidelines on operating room protection, and none of the 12 hospitals had operating room X-ray protection supervisors. Due to the lack of relevant protection guidelines and head of protection and supervision, operating room X-ray protection is not standardized, such as $64 \%$ of the operating room in the X-ray film ignored the patient's non-irradiated parts of the protection. Although China's overall prevention standards and guidelines are more perfect, but the specific implementation of the status quo are different, X-ray irradiation during strict use of protective equipment accounted for $56 \%$, there are still $22 \%$ of health care workers do not use protective clothing, never wear X-ray examination card nurses accounted for $54 \%$. According to the national radiation safety standards, the occupational individual dose limits, systemic effective dose equivalent for consecutive 5 years does not exceed an average of 20 
$\mathrm{m} \mathrm{Sv}$ / year, limbs and limbs does not exceed $150 \mathrm{~m} \mathrm{~Sv} /$ year [2]. Employees who do not wear the $\mathrm{X}$-ray test card can not track the annual dose, and only through testing can understand the distribution of nurses' averaged annual dose. Therefore, it is necessary to wear the X-ray dose test card. In practice, guidelines for radiological protection in operating theaters should be strengthened and refined, and the maneuverability of the guidelines should be tested to ensure that operating room doctors and nurses will regulate X-ray protection and ensure the safety of medical staff and patients.

\section{Discussions}

First, the establishment of radiation equipment operation related systems, including the specific operation of radiation equipment and specific protection system, while monitoring the implementation of health care workers to perform regular checks [2]. Second, to promote the improvement of the hardware facilities in medical facilities. If the hospital needs to receive medical services such as interventional therapy, orthopedic surgery and contrast imaging, such operating rooms must be equipped with special operating rooms for shielding the radiation and the area must be $25 \mathrm{~m} 2$. In addition, according to the workload of surgery also equipped with sufficient protective equipment, including protective clothing, hats, neck circumference and protective lead screen [3]. Third, to promote the improvement of the awareness of protection for medical staff, we must first organize health care workers to learn about the damage caused by radiation on the body in order to improve the compliance of medical staff to wear protective equipment. In addition to strengthen the protection of health care workers targeted training, medical staff before using radiation equipment must be targeted training of professionals, only trained personnel to allow the operation of radiation equipment. Health care workers must follow the shielding, time and distance in the three principles, to develop a good specific procedure for the use of radiological equipment, targeted to develop protective guidelines to monitor the actual implementation of medical staff [4]. For medical personnel who need close contact with the radiological equipment, they must wear a lead rubber cap larger than $0.35 \mathrm{~mm}$ lead equivalent and a high-lead lead apron, or choose to stand behind the lead screen. If a health care provider needs to accompany a patient while working on a radiological equipment, mobile shielding facilities need to be provided for health care workers for a long time, and protective clothing must be worn if the health care provider must work in a radiation area. The most important consideration in the purchase of radiological equipment in medical settings is to minimize the radiation exposure to healthcare professionals and patients. Fourth, the management of medical facilities to do radiation equipment and protective equipment for regular testing, detection of problems found in the medical field should be promptly resolved. Radiological protection around the wall panels of the special operating room has been taken into consideration since the beginning of the design. The top and bottom of the floors are made of cast-in-place concrete, and the electric sliding door in the operating room also adopts the lead with good protection effect. Flow surgery itself there is a positive pressure, and the operating room purification unit has enough air flow, which is conducive to the spread of harmful gases generated by ionizing radiation; can better meet the needs of ventilation.

\section{Conclusions}

This article understands the current situation of radiological protection in the operating room of medical institutions and puts forward some countermeasures for the existing defects in the status quo. Methods the questionnaire was issued to the operating room of 21 medical institutions operating in Zhongshan City to obtain the actual status of intraoperative radiological protection. Results in the operation rooms of 21 medical institutions, there were 24 mobile radiological equipment in 19 medical institutions 'operating rooms and 14 radiological protective clothing in 14 medical institutions' operating rooms. Only $7.37 \% .42 \%$ of nurses did not wear protective clothing during operation, and more than $60 \%$ of nurses did not wear protective clothing while performing radiological operations, and left the operating room or stood behind the protective screen to avoid 
the radiation. All operating room surveyed nurses have not received any professional training in radiological protection. Conclusion Intraoperative radiological protection facilities in medical institutions are equipped and used more freely. The lack of specific knowledge of operatives and improper protection methods should be paid more attention and improvement.

\section{References}

[1] Gao Chengjie, Xie Lianfeng. Operation of postoperative disinfection of the hospital operating room [J]. Prevention medicine literature information. 2001 (06):106-109.

[2] Xu Xiuqin. Grassroots hospital operating room nurses occupational exposure prevention [J]. Chinese Journal of Hospital Infectious Diseases. 2011 (08):29-30.

[3] Liu Xinyu hospital operating room disinfection monitoring status quo and analysis [J]. World's newest Medical Information Digest. 2015 (65):56-58.

[4] Li Yuchuan. Comprehensive hospital operating room infection related factors and control [J]. Qinghai Medical Journal. 1999 (02):87-89.

[5] Zhuang Jing, Wang Zhiqiang, Zhang Huixia, Wang Jirui. County hospital operating room infection causes [J]. Medical Animal Control. 2002 (12):211-212.

[6] Fu Xiurong, Yang Hui, Kang Fengying, Shi Meixia. SARS ward living area protection and management of medical staff [J]. Nursing Research. 2003 (17):97-99.

[7] Bao Jianzhong, Chen Xiulan, Zhai Jianqing, Cao Hong. Safety operation and protection management of $3.7 \times 1015 \mathrm{~Bq} \gamma$ irradiation device [J]. Chinese Journal of Radiological Health, 2003 (04):76-81. 